\title{
Ueber die unmittelbare Wirkung des Accelerans und Vagus auf automatisch schlagende Abschnitte des Säugethierherzens.
}

\author{
II. Mittheilung ${ }^{1}$, zugleich III. Mittheilung ${ }^{2}$ ) \\ über die Erregungsleitung im Säugethierherzen. \\ Von
}

Prof. H. E. Hering (Prag).

(Mit 2 Textfiguren und Tafeln V-IX.)

Bevor ich im Folgenden weitere Versuche über die unmittelbare Wirkung des Accelerans und Vagus auf die automatisch schlagenden Kammern des Säugethierherzens mittheile, muss ich gewisser Versuche von Bayliss und Starling ${ }^{3}$ ) aus dem Jahre 1892 Erwähnung thun, die am Säugethierherzen Beobachtungen gemacht haben, auf Grund welcher sie annahmen, dass der Vagus und der Accelerans die Frequenz der Ventrikel des Säugethierherzens direct ändern könne. Ich selbst ${ }^{4}$ ) habe auf diese Versuche im Jahre 1901 die Aufmerksamkeit gelenkt (denn es wird ihrer in keinem mir bekannten physiologischen Lehrbuch und in keiner mir bekannten Abhandlung Erwähnung gethan), was mir jedoch wieder aus dem Gedächtniss gekommen war; sonst hätte ich diese Versuche schon in der I. Mittheilung ${ }^{5}$ ) erwähnt.

Von der Deutung der Versuche von Bayliss und Starling habe ich schon damals gesagt, dass sie nicht einwandsfrei sei, dass die Beobachtungen sehr kurz und nicht verständlich genug geschildert sind, und dass die diesbezüglichen Curven fehlen.

Bayliss und Starling haben auf einer Tafel zwei Curven

1) Siehe dieses Arch. Bd. 107 S. 125.

2) Siehe die vorangehende Mittheilung.

3) Journ. of Physiol. vol. 13 p. 407. 1892.

4) Dieses Archiv Bd. 86 S. 559. 1901.

5) Dieses Archiv Bd. 107 S. 125. 1905. 
abgebildet, welche andere Thatsachen veranschaulichen, die aber nicht entfernt an die Bedeutung jener Frage heranreichen, ob die Herznerven die Frequenz der Ventrikel des Säugethierzens direct ändern können. Warum sie gerade diese Curven nicht veröffentlicht haben, welche viel wichtiger gewesen wären, ist mir nicht bekannt.

Bezüglich des frequenzändernden Vaguseinflusses auf die Kammern berichten Bayliss und Starling auf S. 411 nur Folgendes:

"There is no doubt however that the ventricles are much less subject to vagus influence than the auricles. The direct action of the vagus on the ventricles can be indubitably shown when, under moderate stimulation, the normal rbythm is reversed, and the auricles are beating extremely feebly after the ventricles. Instances of this we have observed a few times, and have then noticed that strengthening the vagus stimulation causes further slowing or stopping of the ventricular beat and the same result is invariably obtained in the common case of apparent complete auricular inhibition."

Da Bayliss und Starling in dieser Mittheilung sich der Methode bedient haben, durch elektrische Reizung der Ventrikel diesen und durch Rückläufigkeit auch den Vorhöfen einen künstlichen Rhythmus aufzuzwingen, ist es nicht klar; ob in dem oben erwähnten Fall (when the normal rhythm is reversed) die Schlagumkehr eine natürliche oder künstliche war. Da ferner die Vorhọ̆fe extrem schwach schlugen, ist es nicht unwahrscheinlich, dass von ihnen zu dieser Zeit keine entsprechenden Curven zu erhalten waren, die Erscheinung also vielleicht nur beobachtet, aber nicht verzeichnet wurde. Wäre die Schlagumkehr eine künstlich erzeugte gewesen, dann würden diese Versuche darauf hinweisen, dass die Anspruchsfähigkeit der Ventrikel bei Vagusreizung sich vermindern kann, nicht aber, dass ventriculäre Ursprungsreize durch den Vagus beeinflusst werden können.

Ist die Schlagumkehr eine natürliche gewesen, dann würde es sich noch um die Grösse des Intervalles $V_{s}-A_{s}$ handeln, denn es käme noch die Automatie der Verbindungsfasern in Betracht.

Bezüglich der kurzen Darstellung der "one or two cases“, in welcher Bayliss und Starling eine directe frequenzändernde Wirkung des Accelerans auf die Kammern beobachtet zu haben glauben, verweise ich auf das, was ich ${ }^{1}$ ) S. 560 seiner Zeit gesagt habe.

1) Dieses Archiv Bd. 86. 1901. 
Jetzt, nachdem ich meine Beobachtungen gemacht habe, stehen die Deutungen der Beobachtungen von Bayliss und Starling allerdings in einem anderen Lichte da, und man muss Bayliss und Starling wenigstens das Verdienst zuschreiben, an eine Auffassung ihrer Beobachtungen gedacht zu haben, mit der man in der Herzphysiologie nicht gerechnet hat. Dieses Verdienst bleibt bestehen, auch wenn man in Betracht zieht, dass sie sich der grossen Bedeutung jener Auffassung damals nicht bewusst waren; sonst hätten sie gewiss durch weitere Verfolgung jener Beobachtungen und durch Veröffentlichung entsprechender Curven die Richtigkeit ihrer Auffassung auch nachzuweisen gesucht, was sie bis jetzt, soviel mir bekannt ist - und es sind seit jener Zeit 13 Jahre verflossen nicht gethan haben.

\section{Ueber die unmittelbare Wirkung des Accelerans auf die auto- matisch schlagenden Kammern des Affenherzens.}

Nachdem ich mich in zahlreichen Versuchen davon überzeugt hatte, dass der Accelerans die Schlagzahl der automatisch schlagenden Kammern des Hundeherzens, des Katzenherzens und des Kaninchenherzens unmittelbar unter gleichzeitiger Verstärkung ihrer Contractionen zu steigern vermag, bestand für mich kein Zweifel, dass sich das Affenherz ebenso verhalten würde, und in der That wurde meine . Voraussetzung auch völlig bestätigt.

Versuch vom 1. Februar 1905. Ein Kronenaffe wurde in der Aethernarkose verblutet und die Kammern, nachdem vorher die Vorhöfe an der Atrioventriculargrenze weggeschnitten worden waren, durch Speisung 'mit Ringer'scher Lösung zum Schlagen gebracht.

Acceleransreizung hatte unmittelbare Beschleunigung und Verstärkung der Ventrikelcontractionen zur Folge; die Ventrikel schlugen automatisch; Fig. 1 und 2 zeigt die Acceleranswirkung und das Verhalten der ventriculären Extrasystolen.

Die Acceleranswirkung wurde, da die Vorhöfe weggesehnitten waren, durch diejenigen Nerven bewirkt, welche in der Gegend der grossen Gefässe - Aorta und Pulmonalis - zum Herzen ziehen.

\section{Ueber die gleichzeitige Wirkung der Herznerven anf die dissociirt schlagenden Vorhöfe und Kammern.}

Die folgenden Versuche, welche, mit Ausnahme der am Kaninchen gemachten Experimente, an isolirten, mit Ring er'scher Salzlösung 
gespeisten Hundeherzen ausgeführt ;wurden, ergaben alle die unmittelbare. Wirkung des Accelerans auf die Kammern; die Versuche zeigen aber noch viel mehr, und das ist der Grund, sie besonders mitzutheilen.

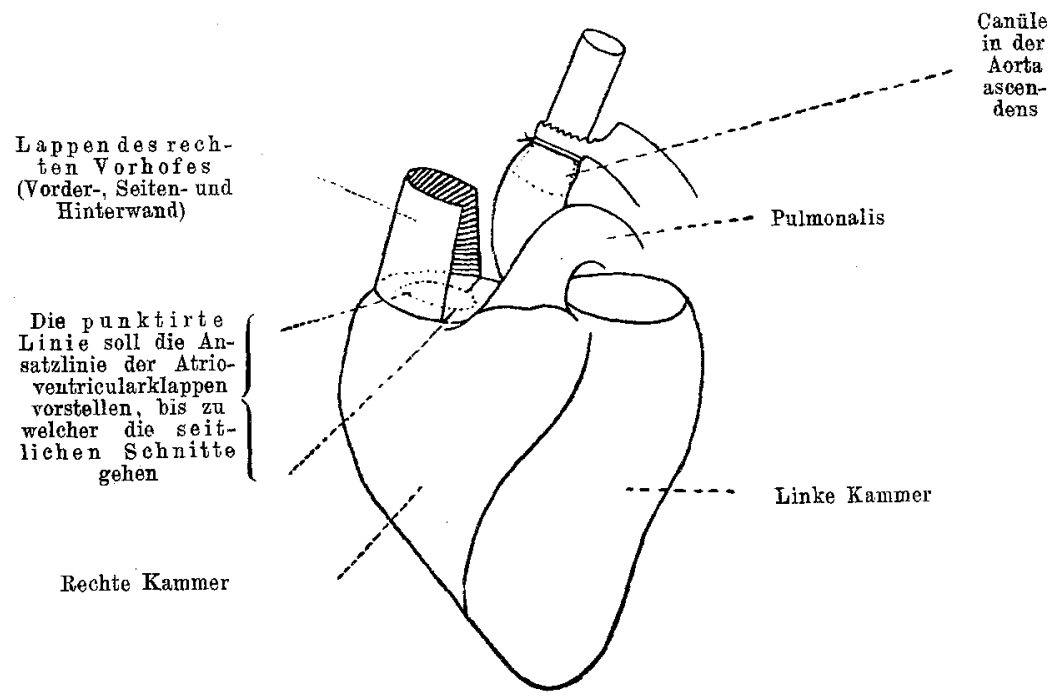

Fig. $A$.

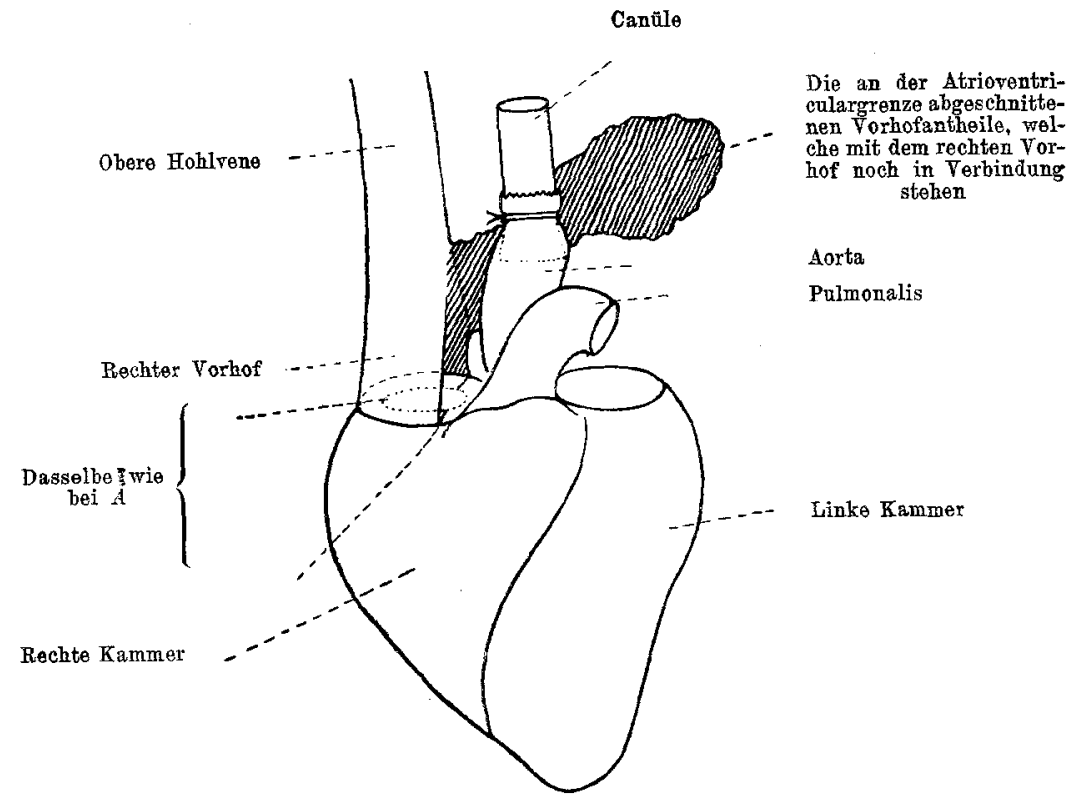

Fig. $B$. 
Damit die am Hundeherzen von mir ausgeführten Versuche leichter verständlich werden, gebe ich im Vorstehenden zwei Abbildungen, Fig. $A$ und Fig. $B$, welche ganz schematisch das Wesentliche der Versuchsanordnung am Herzen wiedergeben. Soweit sich die weiter unten beschriebenen Versuche auf die Durchschneidung des Uebergangsbündels beziehen, verweise ich auf die dieser vorangehende Mittheilung.

Die Abbildung der Figur $A$ soll Folgendes veranschaulichen. An der Atrioventriculargrenze ist von den Vorhöfen mit Einschluss ihrer Scheidewand Alles weggeschnitten bis auf den gezeichneten rechten Vorhoflappen. Um diesen musculär zu isolieren, werden die seitlichen Schnitte gemacht, welche bis zur Ansatzlinie der Atrioventricularklappen reichen. Bei dieser Versuchsanordnung schlägt der rechte Vorhoflappen dissociirt von den beiden Kammern. Das Herz steht nur noch durch das entlang der Aorta und Pulmonalis sich erstreckende Gewebe mit dem Körper in Verbindung, so dass Reizung des Accelerans oder Vagus nur noch auf diesem Wege ihre Wirkung auf das Herz entfalten können.

Fig. $B$ unterscheidet sich von Fig. $A$ nur dadurch, dass der musculär, wie oben, durch seitliche Schnitte isolirte rechte Vorhof noch mit der oberen Hohlvene und den an der Atrioventriculargrenze abgeschnittenen übrigen Vorhofantheilen in Zusammenhang steht, so dass auf diesem Wege die Reizung des Accelerans oder Vagus auf das Herz ihre Wirkung entfalten kann, während die Verbindung des Herzens mit dem Körper durch das Gewebe entlang der grossen Gefässe, Aorta und Pulmonalis, vollständig aufgehoben ist. Es hängt demnach das Herz nur durch den rechten Vorhof mit dem Körper zusammen.

Versuch vom 3. Februar 1905. Hund in der Aethernarkose verblutet. Herstellung eines Kammerpräparates mit isolirten Vorboflappen; darunter ist, wie ich ${ }^{1}$ ) dies früher beschrieben habe, zu verstehen, dass an der Atrioventriculargrenze Alles weggeschnitten wurde bis auf den aus der Vorder-, Seiten- und Hinterwand bestehenden rechten Vorhof, welcher nur mit der rechten Kammer in anatomischem Zusammenhang steht, von ihr aber functionell getrennt ist. (Siehe Fig. A.)

Kammern und Vorhoflappen schlagen dissociirt; die Kammern schlagen automatisch, ebenso der Vorhoflappen. Reizt man jetzt die Herznerven, so können nur diejenigen zur Wirkung kommen, welche in der Gegend der Aorta und Pulmonalis zum Herzen ziehen, denn nur hier besteht noch eine Verbindung des Herzens mit dem übrigen Körper. Wie man aus Fig. 3 und 4 ersieht, hatte Reizung

1) Dieses Archiv Bd. 107 S. 97. 1905. 
des rechten Accelerans zur Folge, dass die dissociirt schlagenden Herzabschnitte (die Kammern und der rechte Vorhof) beschleunigt und verstärkt schlagen.

Abgesehen von diesem functionellen Ergebniss, zeigt dieser Versuch auch, dass Acceleransfasern rückläufig auf den rechten Vorhof übergehen, und zwar handelt es sich um Acceleransfasern, welche, in der Atrioventricularfurche verlaufend, Zweige an den rechten Vorhof abgeben.

Versuch vom 4. Februar 1905, Vormittag. Bei diesem: Experimente wurde der rechte Vorhof ebenfalls functionell von den Ventrikeln isolirt, jedoch die an der Atrioventriculargrenze abgeschnittenen Vorhofantheile mit dem von den Ventrikeln functionell isolirten rechten Vorhof in Verbindung gelassen, so dass entlang der mit dem rechten Vorhof zusammenhängenden Vorhofantheile auch die Verbindung der Herznerven mit dem Herzen noch erhalten war. Damit nun die Herznerven nur auf diesem Wege ihre Wirkung auf das Herz entfalten könnten, wurde der Zusammenhang des Herzens mit dem Körper in der Gegend der grossen Gefässe vollständig aufgehoben. (Siehe Fig. B.)

Das Herz stand also mit dem Körper nur durch den, functionell von den Kammern isolirten, rechten Vorhof vermittelst der mit ihm in Zusammenhang belassenen, an der Atrioventriculargrenze abgeschnittenen übrigen Vorbofantheile in Verbindung.

Rechter Vorhof und die Kammern schlagen dissociirt und automatisch.

Reizung des rechten Vagus + Accelerans bei R.-A. 8 ergab, siehe Fig. 5, nur eine Wirkung auf den rechten Vorhof. Die Wirkung bestand darin, dass die Vorhofcontractionen zunächst abgeschwächt wurden, die noch abgeschwächten Contractionen allmählich etwas häufiger wurden, worauf, nach Sistirung der Reizung, die Acceleranswirkung - beschleunigte und verstärkte Contractionen des Vorhofes - allein zum Ausdruck kamen. Eine etwas stärkere Reizung bei R.-A. 6 ergab vielleicht auch eine ganz geringe Acceleranswirkung auf die Kammern; siehe Fig. 6.

Man kann es bei dieser Versuchsanordnung dahin bringen, dass die Wirkung der extracardialen Herznerven sich nur auf den rechten Vorhof, nicht aber auf die Kammern erstreckt. (Siehe auch weiter unten Versuch vom 20. März 1905.) 
Versuch vom 4. Februar $1905, \mathrm{Nachmittag.} \mathrm{Dieser} \mathrm{in}$ derselben Weise wie der vorausgebende, angestellte Versuch ergab eine Acceleranswirkung nur auf den rechten Vorhof; siehe Fig. 7.

\section{Reizung der extracardialen Herznerven nach Durchschneidung des Uebergangsbündels.}

Von den zehn in dieser Weise angeführten Versuchen seien nur die folgenden zwei erwähnt:

Versuch vom 21. Februar 1905. Es handelt sich hier um jenen Versuch, der in der vorausgehenden Mittheilung schon angeführt ist, in welchem das Uebergangsbündel durchschnitten worden war.

Unmittelbar im Anschluss an Fig. 2 der vorausgehenden Mittheilung wurde Fig. 8 gewonnen. Reizung des rechten Accelerans bei R.-A. 6 ergab eine Wirkung auf die Vorhöfe und Kammern, nur war die Wirkung auf die Kammern relativ sehwach.

Der linke Accelerans wirkte in diesem Falle auf die Kammern stärker als der rechte, wie man aus Fig. 9 und 10 ersehen kann.

Versuch vom 1. März 1905 . Dieser Versuch wurde gleichfalls in der vorausgehenden Mittheilung schon erwähnt. Es handelt sich auch hier um die Durchschneidung des Uebergangsbündels. Reizung des rechten Vagus + Accelerans bei R.-A. 3 ergab uur eine Vagus- + Acceleranswirkung auf den Vorhof; siehe Fig. 11.

Stärkere Reizung des rechten Vagus + Accelerans bei R.-A. 6 ergab ausgesprochene Beschleunigung mit Verstärkung der Vorhöfe wie der Kammern; siehe Fig. 12.

Nun wurde in der Gegend der grossen Gefässe der Zusammenhang des Herzens mit dem übrigen Körper an dieser Stelle aufgehoben; nachträglich zeigte es sich, dass nicht Alles an dieser Stelle durchschnitten war; der undurchschnittene Theil war aber nicht sehr gross.

Reizung des linken Accelerans bei R.-A. 5 ergab nur eine Wirkung auf die Ventrikel; siehe Fig 13.

Reizung des rechten Accelerans bei R.-A. 4 ergab eine Wirkung auf die Vorhöfe und Ventrikel, welche aber schwächer war als in Fig. 11 bei R.-A. 6; siehe Fig. 14 . 


\section{Versuche an nicht isolirten Herzen von Kaninchen.}

Da man beim Abschnüren der Vorhöfe in der Gegend der Atrioventriculargrenze beim Hund leicht Flimmern des Herzens bewirkt und dieses Flimmern; beim Arbeiten am ganzen Thiere nicht $\mathrm{zu}$ beseitigen ist, habe ich zu den folgenden Versuchen vorläufig nur Kaninchen benützt.

Kaninchenversuch am 26. März 1904. Nach Curarisirung des Thieres wurden in der Gegend der Atrioventriculargrenze die Vorhöfe mit einem geölten, nicht zu dünnen Bindfaden so abgeschnürt, dass die Erregungsleitung zwischen den Vorhöfen und Kammern nach Lösung der Schnürung dauernd aufgehoben blieb. Reizung der durchschnittenen Vagi batte nun einen verschiedenen Erfolg auf die Vorhöfe und auf die dissociirt von diesen schlagenden Kammern, wie dies z. B. aus den Curven der Fig. 15 und 16 zu ersehen ist.

Suspendirt war der rechte Vorhof und der rechte Ventrikel. Auch hatte der rechte Vagus eine etwas andere Wirkung als der linke, indem der rechte Vagus bei derselben Reizstärke einen stärkeren Einfluss auf die Vorhöfe hatte als der linke.

Der Grund aber, warum ich diese Versuche hier anführe, ist der, eine unmittelbar frequenzändernde Vaguswirkung auf die unterhalb der Schnürurche gelegenen dissociirt von den oberhalb der Schnürfurchen gelegenen Herztheilen schlagenden Herzabschnittezuzeigen.

An der Vorhofeurve sind die passiv von den Kammern mitgetheilten Bewegungen mit $\mathcal{V}$, die Vorhofcontractionen mit $A$ bezeichnet, so dass man die Dissociation leicht überblicken kann.

Die Vaguswirkung auf die unterhalb der Schnürfurche liegenden Herzabschnitte erfolgte vermittelst jener Vagusfasern, welche in der Gegend der Aorta und Pulmonalis (welche beide nicht mit abgeschnürt wurden) zu den Kammern ziehen.

Das Verbältniss der $A$-Frequenz zu der der $V$-Frequenz verhielt sich ungefähr wie $3: 2$, d. h. es schlugen die Vorhöfe drei Mal, die Kammern ein wenig mehr als zwei Mal pro Secunde, also die Kammern seltener als die Vorhöfe, wie dies bei Dissociation zwischen $A$ und $V$ zu sein pflegt.

Es sei hier noch erwähnt, dass man eine directe Wirkung der Herznerven auf die Kammern auch dann erhalten kann, wenn die Vorhöfe flimmern. 
Die angeführten Versuche, welche nur einige wenige derjenigen sind, welche ich gemacht habe, zeigen Folgendes:

Unabbängig von einander schlagende Abschnitte des Säugethierherzens werden unabängig von einander unmittelbar durch die centrifugalen Herznerven beeinflusst, und zwar nicht nur bezüglich ihrer Contractionsstärke, sondern auch bezüglich ihrer Contractionsfrequenz.

Es ergibt sich weiter aus diesen Versuchen:

Die extracardialen Fortsetaungender extracardialen centrifugalen Herzerven vermitteln die Erregungsleitung zwischen den Vorhöfen und den Kammern nicht, wenn die musculäre Verbindung zwischen den Vorhöfen und den Kammern aufgehoben ist.

\section{Die Erregungsleitung im Säugethierherzen ist eine musculäre.}

Aus den in dieser Mittheilung und aus den in den vorausgegangenen Mittheilungen veröffentlichten Thatsachen lässt sich unschwer ableiten, dass die Erregungsleitung im Säugethierherzen eine musculäre ist.

1. Wenn die musculäre Verbindung zwischen den Vorhöfen und Kammern fehlt, findet keine Erregungsleitung statt.

2. Trotz fehlender musculärer Verbindung und damit fehlender Erregungsleitung zwischen den Vorhöfen und den Kammern ändert die Reizung der extracardialen Herznerven die Stärke der Kammercontractionen principiell ebenso wie bei bestehender musculärer Verbindung und damit bestehender Erregungsleitung.

Es ist nun eine lang bekannte und leicht zu demonstrirende Thatsache, dass bei bestehender Erregungsleitung zwischen den Vorhöfen und Kammern die Stärke der Kammercontractionen unabhängig ist von der Stärke der sie auslösenden Vorhofeontractionen. Dies gilt auch dann, wenn man die Stärke der Vorhofcontractionen durch Reizung der Herznerven ändert.

F. B. Hofmann ${ }^{1}$ ) hat am Froschherzen beobachtet, dass bei spontaner Schlagfolge nach Durchschneidung der Scheidewandnerven trotz der erhaltenen schwächenden Vaguswirkung auf den Vorhof keine Spur einer directen schwächenden Wirkung des Vagus auf den Ventrikel nachweisbar ist.

1) Dieses Archiv Bd. 72 S. 409.1898. 
Dasselbe lässt sich in ähnlicher Weise beim Säugethierherzen zeigen. Es genügt folgenden Versuch anzuführen, welcher sich auf die verstärkende Wirkung des Accelerans bezieht.

Versuch vom 20. März 1905. Die Versuchsanordnung ist fast ganz dieselbe, wie jene bei den Versuchen am 4. Februar 1905 Vormittags und Nachmittags, nur mit dem Unterschiede, dass die Erregungsleitung zwischen dem rechten Vorhof und den Ventrikeln nicht aufgehoben ist. Das Herz hängt, wie erwähnt, nur durch den rechten Vorhof, und zwar durch seine Vorder-, Seiten- und Hinterwand mit dem Körper zusammen. Also nur dadurch, dass man $\mathrm{nicht}$, wie in jenen Versuchen, durch seitliche bis zum Ansatz der Atrioventricularklappen reichende Einschnitte die musculöse Verbindung des rechten Vorhofes mit den Kammern aufhebt, unterscheidet sich diese Versuchsanordnung von jenen beiden Experimenten vom 4. Februar.

Wie aus jenen Experimenten zu ersehen ist, kann man bei dieser Versuchsanordnung erreichen, dass die zu den Kammern ziehenden Acceleransfasern ausgeschaltet werden, so dass Acceleransreizung nur eine Wirkung auf den rechten Vorhof, aber nicht mehr auf die Kammern hat. In jenen Fällen schlug der rechte Vorhof dissociirt von den Kammern. Hebt man aber bei der gleichen Versuchsanordnung die Ueberleitung vom rechten Vorhof $\mathrm{zu}$ den Kammern nicht auf, so dass keine Dissociation, sondern normale Succession zwischen $\mathrm{A}$ und $\mathrm{V}$ besteht, und reizt den Accelerans, dann tritt eine Beschleunigung und Verstärkung der Contractionen des rechten Vorhofes und eine vom Vorhof abhängige Beschleunigung der Kammercontractionen ein, aber die Verstärkung der Kammercontractionen bleibt aus, letztere werden vielmehr in Folge der Beschleunigung kleiner; siehe Fig. 17.

Dasselbe lässt sich für die schwächende Vaguswirkung zeigen, und zwar beim Säugethierherz insofern noch leichter als beim Froschherz, weil die schwächende Vaguswirkung sich an den Kammern der Säugethierherzen im Allgemeinen überhaupt schwächer auszuprägen pflegt, als beim Frosehherzen.

Aus diesen Thatsachen ergibt sich Folgendes:

Die Aenderungen in der Stärke der Vorhofcontractionen des Säugethierherzens haben keine entsprechenden Aenderungen in der Stärke der 
Kammercontractionen zur Folge, und zwar auch dann nicht, wenn die Stärke derVorhofcontractionen dureh Nerveneinfluss geändert wird.

Allgemein gesagt:

Aenderungen in der Contractionsstärke eines Herzabschnittes werden beim Säugethier nicht durch Leitung, sondern durch unmittelbare Einwirkung der jeweiligen Ursache auf den betreffenden Herzabschnitt hervorgerufen.

Diejenigen, welche ein intramusculäres Nervennetz annehmen, werden zugeben, dass gar kein Anhaltspunkt dafür vorliegt, dass das intramusculäre Nervennetz, falls es überhaupt vorhanden ist, mit den intracardialen Fortsetzungen der extracardialen centrifugalen Herznerven keinen anatomischen und physiologischen Zusammenhang habe.

Stellen wir uns einmal auf den Standpunkt der neurogenen Theorie der Herzthätigkeit, und nehmen wir an, die Erregungsleitung im Herzen wäre eine nervöse: dann müssten auch die Herzreize nervöse sein. Nun wissen wir, dass unter dem Einfluss der extracardialen centrifugalen Herznerven das Herz zu einer selteneren oder häufigeren Sehlagfolge veranlasst werden kann. Es würden also z. B. bei Acceleransreizung mehr nervöse Reize gebildet werden, deren Erregungen auf nervösem Wege weitergeleitet würden. Die Acceleransreizung hat nun aber immer, wenn sie eine Vermehrung der Schlagzahl bewirkt, auch eine Verstärkung der Contractionen zur Folge.

Aus den zuletzt oben erwähnten Versuchen geht nun hervor, dass nach Ausschaltung des Einflusses der extracardia len Acceleransfasern auf die Kammern die Acceleransreizung auf den Vorhof zwei Wirkungen - Beschleunigung und Verstärkung auf die Kammer aber nur eine Wirkung hat - Beschleunigung; und zwar hat sie auf den Ventrikel desswegen nur eine Wirkung, weil Stärkeänderungen der Contractionen eines Herzabschnittes nicht durch Leitung bewirkt werden.

Da nun Stärkeänderungen der Contractionen eines Herzabschnittes nicht durch Leitung bewirkt werden, kann es im Herzen auch keine nervöse Leitung für die durch die extracardialen centrifugalen Herznerven be- 
wirkten Stärkeänderungen der Contractionen eines Herzabschnittes geben.

Wenn es also überhaupt eine nervöse Leitung im Säugethierherzen gibt, dann kann diese nervöse Leitung nur dazu dienen, die Frequenzänderungen der Contractionen eines Herzabschnittes anderen Herzabschnitten mitzutheilen.

Ich habe nun festgestellt, dass Frequenzänderungen der Kammern unter dem Einfluss der extracardialen Herznerven auch dann erfolgen, wenn die Erregungsleitung zwischen den Vorhöfen und den Kammern aufgehoben ist, oder mit anderen Worten: Die unter dem Einfluss der extracardialen centrifugalen Herznerven erfolgenden Frequenzänderungen eines Herzabschnittes werden durch directe Wirkung der Herznerven auf den betreffenden Herzabsehnitt bervorgerufen. Die frequenzändernde Wirkung der Herznerven kommt demnach ebenso wie ihre contractionsändernde Wirkung zu Stande, d. h. durch unmittelbare Wirkung der betreffenden Herznerven auf den jeweiligen Herzabschnitt. Für die contractionsändernde Wirkung gibt es keine nervöse Leitung. Warum sollen wir nun für die frequenzändernde Wirkung eine nervöse Leitung annehmen, da die extracardialen Herznerven diese Wirkung ebenfalls unmittelbar erzielen und das Herz ein anderes Gewebe besitzt, welches leitet, nämlich das Muskelgewebe? Es liegt demnach nicht nur keine Nötigung zu der Annahme vor, die mittelbar ausgelösten Frequenzänderungen eines Herzabschnittes würden durch nervöse Leitung bewirkt, sondern es sprechen gewisse Thatsachen nicht dafür, und wir können die mittelbar ausgelösten Frequenzänderungen, ohne in Widerspruch mit den vorhandenen Thatsachen zu gerathen, mit Hilfe der musculären Leitung erklären.

Ich komme weiter unten noch auf den Umstand zurück, dass es wahrscheinlich nur zwei Nervenarten gibt, und dass auch dieser Umstand gegen die nervöse Leitung im Herzen spricht. Ziehen wir jetzt in Betracht, dass die Erregungs ü b e r leitung zwisehen $A$ und $V$ ein Muskelbundel vermittelt.

Da es nun festgestellt ist, dass die Erregungsüberleitung ein Muskelbündel vermittelt, können wir dem hypothetischen intramusculären Nervennetz, welches sich natürlich auch auf das Muskelbündel erstrecken müsste, die Erregungsüberleitung nicht zuschreiben, denn man wüsste wahrlich nicht, was die Muskelfasern dieses Muskelbündels dann für eine Function besitzen sollten. Auch 
wäre, wenn nicht die Muskelfasern, sondern das in ihnen angenommene Nervennetz die Erregungsüberleitung vermitteln würde, das Uebergangsbündel, wie die neurogene Theorie angenommen hat, anscheimend überflüssig.

Da das Uebergangsbündel sicher die Function hat, die Erregung überzuleiten, so würde es, wollte man in demselben noch ein leitendes Nervennetz annehmen, eine doppelte Leitung im Herzen geben, eine nervöse und eine musculäre: eine Annahme, welche ebenso überflüssig erschiene als jene, dass die Ueberleitung nur auf dem Wege eines intramusculären Nervennetzes erfolge, nachdem es erwiesen ist, dass ein Muskelbündel die Verbindung zwischen den Vorhöfen und den Kammern herstellt.

Man bedenke ferner Folgendes:

Wenn ein intramusculäres Nervennetz vorhanden wäre, welches die Leitung zwischen Vorhof und Kammer vermitteln würde, wozu wären dann die vielen Nerven vorhanden, welche ausserhalb des Uebergangsbündels zur Kammer ziehen? Dass diese Nerven nicht etwa nur centripetale sind, beweisen zur Genüge meine Versuche. Es sind das jene Nerven, welche bis zur Kammer extramusculär laufen, um erst in der Kammer mit der Musculatur in Verbindung zu treten, d. h. dort, wo sie ihre Wirkung unmittelbar. entfalten.

Durch meine Versuche ist erwiesen, dass selbst, wenn ein intramusculäres Nervennetz im Vorhof und in der Kammer vorhanden ist, das Nervennetz von $A$ mit dem Nervemnetz von $V$ durch jene centrifugalen Nerven, welche ausserhalb des Uebergangsbündels zur Kammer ziehen, in keiner functionellen Verbindung steht.

Es ist damit auch jener Ausweg, den ich für die Anhänger der nervösen Leitung auf S. 103-104 dieses Archives Bd. 107 angedeutet hatte, erledigt.

Für die Anhänger der nervösen Leitung im Herzen wüsste ich nach den Ergebnissen meiner letzten Experimente beim besten Willen keinen Ausweg mehr. Die Annahme einer nervösen Leitung: im Säugethierherzen wäre jetzt, nachdem es erwiesen ist, dass sowohl die stärkeändernde als auch die frequenzändernde Wirkung der extracardialen centrifugalen Herznerven durch unmittelbaren Einfluss auf jene Herzabtheilungen zu Stande kommen, zu welchen sie auf extramusculärem Wege direct hinziehen, nicht nur überflüssig, sondern es wäre jetzt auch geradezu unverständlich, wie eine solche 
directe Wirkung der Herznerven möglich wäre, wenn eine nervöse leitende Verbindung im Herzen vorhanden wäre. -

Ich $\left.^{1}\right)$ habe schon früher einmal darauf hingewiesen, dass nach meinen Erfahrungen beim Säugethierherzen das Intervall $A_{s}-V_{s}$ eine Sekunde betragen kann. Wäre die Ueberleitung eine nervöse, so wäre diese lange Ueberleitungszeit ein Unicum für das Nervensystem des Sängethieres, denn es gibt kein einziges Beispiel beim Säugethier dafür, dass eine nervöse Leitung so viel Zeit in Anspruch nehmen könne. -

Auf eine Beobachtung sei noch kurz eingegangen, welche ich schon wiederholt in früheren Mittheilungen erwähnt habe, nämlich jene, dass die Vorhöfe anscheinend, ohne sich zu contrahiren, die Erregung auf die Kammern übertragen können, eine Erscheinung, die ich sehr oft beim Säugethiere studirt habe. In allen solchen Fällen habe ich mich davon überzeugen können, dass der auf den Vorhof einwirkende Reiz, wenn er eine Kammercontraction zur Folge hatte, immer eine, wenn auch oft nur relativ schwache und wenig, ausgebreitete Contraction am Vorhof bewirkte.

Diese Beobachtungen sind nur extreme Fälle jener oben erwähnten Erscheinung, dass die Stärke der Kammercontraction unabhängig ist von der Stärke der sie auslösenden Vorhofcontraction.

Während diese Erscheinung lange bekannt ist, fehlte doch eine Erklärung derselben, welche ich $^{2}$ ) im Jahre 1901 gegeben habe, indem ich auf den bis dahin gar nicht beachteten Umstand aufmerksam machte, dass das Gesetz der maximalen Reaction des Herzens nicht nur für die Contraction, sondern auch für die Leitung Geltung hat: ein Umstand, dem nach mir bis jetzt, soviel mir bekannt ist, nur ein Forscher - J. v. Kries ${ }^{3}$ ) noch Beachtung geschenkt hat.

$\mathrm{Da}$ ich bei anderer Gelegenheit auf diesen Umstand, welcher besagt, dass der Herzmuskel auf Leitungsreize ebenso maximal seinem jeweiligen Zustande entsprechend reagirt wie auf den Ursprungsreiz, zurückzukommen gedenke, sei hier auf das hingewiesen, was ich schon im Jahre 1901 über dieses besondere Verbalten der Leitung im Herzmuskel gesagt habe. -

1) Dieses Archiv Bd. 86 S. 573. 1901.

2) Dieses Archiv Bd. 86 S. 550. 1901.

3) Arch. f. Physiol. 1902 S. 490. 
Wessen Gedanken sich noch nicht allzu sehr in dem hypothetischen intramusculären Nervennetze verstrickt haben, der wird zugestehen, dass wir jetzt wohl genügend Grund haben, zu sagen, die Erregungsleitung im Säugethierherzen ist eine musculäre, während für die Annahme einer nervösen Leitung weder ein entsprechender Anhaltspunkt noch irgend eine Nötigung vorhainden ist. -

Dieses Capitel möchte ich nicht schliessen, ohne davor gewarnt $\mathrm{zu}$ haben, eine musculäre Leitung überall da anzunehmen, wo wir bei einem Lebewesen ein Organ finden, das eine dem Herzen ähnliche Function besitzt.

Die Beobachtungen, welche A. J. Carlson ${ }^{1}$ ) bei Limulus gemacht hat, berechtigen sehr zu dieser Warnung. Mehrere seiner Beobachtungen stimmen nicht mit denjenigen überein, welche sich beim Säugethierherzen machen lassen. Es sind zum Theil andere Thatsachen an einem anatomisch und functionell zum Theil andersartigen Organ. Es ist ein grosser Fehler, der leider auch jetzt noch oft genug gemacht wird, die am Herzen von Nicbtsäugethieren - gemachten Beobachtungen auf diejenigen zu übertragen, welche sich am Herzen der Säugethiere machen lassen, ohne sich zu vergewissern, ob man es mit einer principiell gleichartigen Erscheinung an einem principiell gleichartigen Substrat unter principiell gleichartigen Umständen zu thun hat. So darf man z. B. die am Froschherzen zu beobachtende Erscheinung, dass der Sinus bäufiger schlägt als der Vorhof, nicht auf das Säugethierherz übertragen, wie es irrthümlicher Weise öfters geschieht, weil weder die Erscheinung noch das betreffende Substrat beim Säugethierherzen zu finden ist.

Ich bringe daher im Titel meiner Arbeiten nicht unabsichtlich zum Ausdruck, dass es sich um Beobachtungen an Säugethierherzen handeit. Was wir von den beim Experiment an Säugethierherzen gemachten Beobachtungen auf das menschliche Herz übertragen dürfen, ist sehr viel, und ich habe noch keinen einzigen principiellen Unterschied zwischen der Function des menschlichen Herzens und der Function des Herzens jener Säugethiere beobachten können, welche beim Experiment hier zur Verwendung kommen (Affe, Hund, Katze, Kaninchen). Trotzdem übertrage ich die am Säugethierherzen gemachten Beobachtungen nicht auf das menschliche Herz, wenn sich

1) Americ. Journ. of Physiol. vol. 12 Nr. 1. 1904, und Nr. 5. 1905. 
die Umstände nicht vergleichen lassen, unter denen die Beobachtungen bei Thier und Mensch gewonnen wurden ${ }^{1}$ ).

Diejenigen, welche das, was ich soeben gesagt habe, für etwas Selbstverständliches halten, mögen diese Bemerkungen entschuldigen; dass sie nicht überflüssig sind, zeigt zur Genüge die Literatur.

\section{Ueber die Annahme von nur zwei Arten centrifugaler Herznerven.}

Ich habe es immer für genügend angesehen, nur zwei Arten centrifugaler Herznerven zu unterscheiden, wie ich ${ }^{2}$ ) dies auch im Jahr 1901 zum Ausdruck gebracht habe.

Was mich veranlasst, auf diese Annahme zurückzukommen, ist folgender neuer Umstand, welcher mir bei meinen letzten Versuchen aufgefallen ist.

Wirkt der Vagus oder Accelerans auf einenatomatisch schlagenden Herzabschnitt frequenzändernd, dann änderter auch die Stärke seiner Contractionen, während diese Nerven die Contractionen eines nicht automatisch schlagenden Herzabschnittes nur der Stärke nach ändern.

Meine bis jetzt angestellten Beobachtungen lassen mich demnach Folgendes annehmen: Wie der Vagus so wirkt auch der Accelerans immer gleichzeitig frequenzändernd und stärkeäudernd, aber es kann die stärkeändernde Wirkung an einem Herzabschitt allein auftreten, ohne dass die Frequenz des Herzens sich, ändert, wenn dieser Herzabschnitt nicht automatisch schlägt.

Als ich die unmittelbar beschleunigende Wirkung des Accelerans auf die Kammern beobachtete, fragte ich mich gleich (siehe Seite 127

1) Ich habe inzwischen das Glück gehabt, das Herz eines 35 Jahre alten Manues $11 \mathrm{Stunden}$ nach seinem Tode durch Speisung mit Ringer'scher Lösung wieder zu beleben. Ich werde über diesen Fall in der Zeitschrift für experimentelle Pathologie und Therapie berichten. Hier sei nur dasjenige Allgemeinergebniss hervorgeboben, welches mir das Wesentlichste zu sein scheint. Ich habe bei diesem menschlichen Herzen, an welchem ich während drei Stunden die verschiedensten Er. scbeinungen studirte, keine einzige Beobachtung gemacht, welche mir nicht schon vom Experiment am Säugethierherzen bekannt gewesen wäre.

2) Dieses Archiv Bd. 86 S. 574. 1901. 
d. Arch. Bd. 107), warum tritt diese Wirkung normaler Weise, d. b. dann, wenn die Kammern in Abbängigkeit von den Vorhöfen schlagen, nicht in Erscheinung?

Die Antwort war sehr naheliegend: Weil die Automatie des auriculären Herzendes grösser ist als die des ventriculären Herzendes.

Es kann sich wohl kaum anders verhalten als folgendermassen: Wirkt die Erregung des Accelerans oder des Vagus auf das in normaler Succesion schlagende Herz ein und kommt es zu einer Frequenzänderung, dann wirken diese Nerven auch gleichzeitig frequenzändernd auf die Kammern, aber diese Wirkung kann nicht zum Ausdruck kommen; weil die Kammern in Abhängigkeit von einem Herzabschnitt schlagen, dessen Automatie eine grössere ist als die der Kammern.

Nur in jenen abnormen Fällen, in welchen die Automatie des auriculären Herzendes aus irgend einem Grunde darniederliegt, kann auch die frequenzändernde Wirkung an den Kammern in $\mathbf{E r}$ scheinung treten, in welchen Fällen dann die Kammern automatisch schlagen und, wenn es zur Rückläufigkeit der Erregung kommt, auch den Rhythmus des auriculären Herzendes beherrschen.

Aendert der Vagus oder Accelerans nur die Stärke der Contractionen, nicht aber die Frequenz eines Herzabschnittes, dann wirken in diesem Falle die Nerven nicht auch gleichzeitig auf den Herzabschnitt, welcher die Schlagfolge des Herzens bestimmt, oder jener Herzabschnitt ist durch künstliche Reize zum Schlagen angeregt.

So weit ich es aus den in der Literatur vorhandenen Beobachtungen am Froschherzen ersehen kann, dürtten diese Verbältnisse auch für Letzteres Geltung haben. Beim Frosch kann man nach diesen Angaben bei Reizung des Vagus unter Umständen eine stärkeändernde Wirkung nur auf den Vorhof erhalten, aber dieser schlägt beim Froseh normaler Weise nicht automatisch, und er grenzt sich, wie erst oben wieder betont, functionell vom Venensinus $a b$, während dies beim Säugethier nicht der Fall ist.

Keine der bis jetzt am Kaltblüter gemachten Beobachtungen über die Wirkung der extracardialen Herznerven widerspricht direct meiner Auffassung.

Es ist wohl kaum nothwendig, zu erwähnen, dass man bei der Beurtheilung der Stärkeänderungen, wenn sie mit Frequenzänderungen einhergehen, immer den Einfluss der Frequenzänderung auf die Stärkeänderung berücksichtigen muss. - 
Wenn sich nun meine Auffassung weiterhin bestätigen sollte, so würde sich aus derselben noch ein weiterer Umstand gegen die nervöse Leitung im Herzen ergeben, auf den ich weiter oben schon kurz hingewiesen habe.

Wenn die frequenzändernde und die contractionsändernde Wirkung des Accelerans oder des Vagus immer gleichzeitig erfolgt (wenn auch, wie gesagt, beide Wirkungen nicht immer aus erwähnten Gründen gleichzeitig zum Ausdruck kommen) und wir daraus schliessen, dass dieselben Nerven der einen Art beide Wirkungen hervorrufen, dann würde auch dieser Umstand gegen die Annahme sprechen, dass die frequenzändernde Wirkung auf nervösem Wege weiter geleitet würde, da die stärkeändernde Wirkung, wie weiter oben erwähnt, sicher nicht auf nervösem Wege weiter geleitet wird, weil Stärkeänderungen eines Herzabschnittes überhaupt sich nicht durch Leitung einem anderen Herzabschnitte mittheilen.

\section{Erklärung der Figuren 1-17.}

Alle Figuren sind von links nach rechts zu lesen. Die Zeit ist immer in Secunden angegeben. Es wurden (mit Ausnahme in der Fig. 1 und 2, wo nur der rechte Ventrikel verzeichnet wurde) immer die Contractionen des rechten Vorhofes (A) und des rechten Ventrikels (V) nach der Suspensionsmethode verzeichnet. Die faradische Reizung der Nerven, wie die Auslösung der Extrasystolen $(E)$ durch Einzelinductionsschläge erfolgte mit Hilfe von zwei Daniellschen Elementen.

Alle Figuren stammen vom Hundeherzen, mit Ausnahme der Fig. 1 und 2, welche von einem Affenherzen, und der Fig. 15 and 16, welche von einem Kaninchenherzen herrühren.

Fig. 1 und 2 stammt vom Versuch am 1. Februar 1905 und zeigt die Wirkung der Acceleransreizung auf die automatisch schlagenden Kammern des Affenherzens. Bei $E$ wurden ventriculäre Extrasystolen ausgelöst, deren Periodenlänge die Automatie der Kammern zeigt.

Fig. 3 und 4 stammt vom Versuch am 3. Februar 1905 und zeigt die gleichzeitige Wirkung des Accelerans auf die dissociirt schlagenden $A$ und $V$. Bei $E$ wurden ventriculäre Extrasystolen ausgelöst.

Fig. 5 und 6 stammt vom Versuch am 4. Februar 1905 Vormittag, Fig. 7 stammt vom Versuche am 4. Februar 1905 Nachmittag. $A$ und $V$ schlagen dissocirt. In Fig. 6 wurden bei $E$ ventriculäre Extrasystolen ausgelöst. In Fig. 5, 6 und 7 wurde der Vagus + Accelerans gereizt. 
Ueber die unmittelbare Wirkung des Accelerans und Vagus etc. $\quad 299$

Fig. 8, 9 und 10 stammt vom Versuch am 21. Februar 1905 (Durchschneidung des Uebergangsbündels). $A$ und $V$ schlagen dissociirt. Die kleinen Erhebungen in der Kammercurve rühren vom Vorhofe, und die Senkungen in der Vorhofcurve von der Kammer her. Acceleransreizung wirkt auf die Vorhöfe und die Kammern gleichzeitig.

Fig. 11, 12, 13 und 14 stammen vom Versuche an 1. März 1905 (Durchschneidung des Uebergangsbündels). In Fig. 11 wurde der rechte Vagus + Accelerans gereizt, Wirkungen auf $A$; in Fig. 12 Wirkung auf $A$ und $V$; in Fig. 13 ergab Reizung des linken Accelerans nur Wirkung auf $V$; in Fig. 14 ergab Reizung des rechten Accelerans Wirkung auf $A$ und $V$, welche immer dissociirt schlagen. In Fig. 13 bei $E$ Auslösung einer ventriculären Extrasystole.

Fig. 15 und 16 stammen vom Kaninchen (Versuch vom 26. März 1904). In der Vorhofcurve sind auch die passiv mitgetheilten Ventrikelcontractionen sichtbar. $A$ und $V$ schlugen dissociirt; in Fig. 15 wurde der rechte, in Fig. 16 der linke Vagus faradisch gereizt.

Fig. 17 stammt vom Versuche am 20. März 1905. Normale Succession von $\boldsymbol{A}$ und $V$. Bei Reizung des rechten Accelerans erfolgt eine verstärkende Wirkung nur auf $A$. Die Frequenzänderung von $V$ ist die Folge der Frequenzänderung von $A$. 


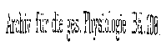

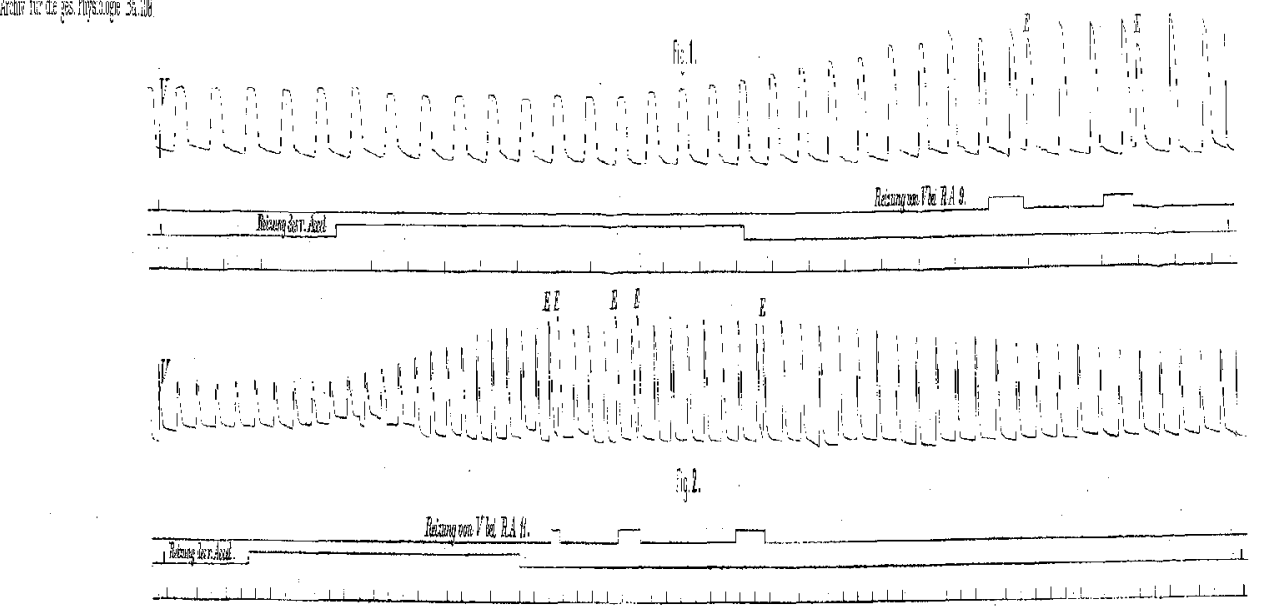

D lute

[in]

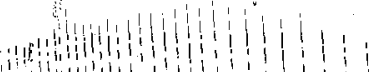
(a)

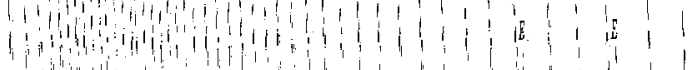

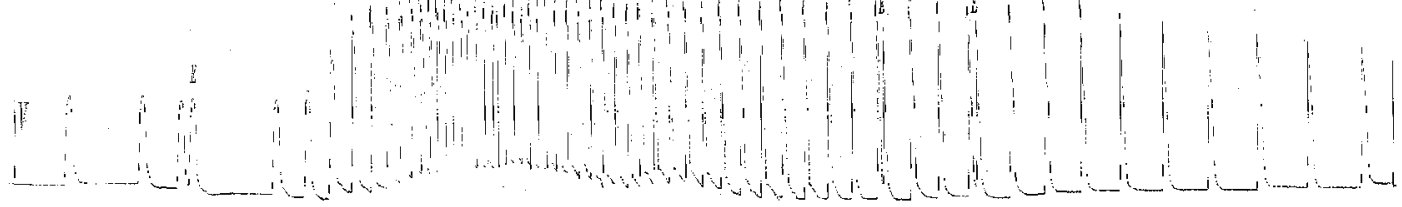

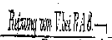

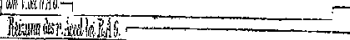

A th

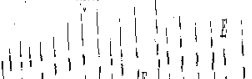

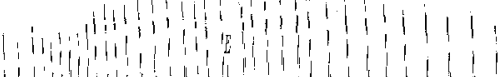

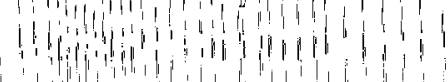

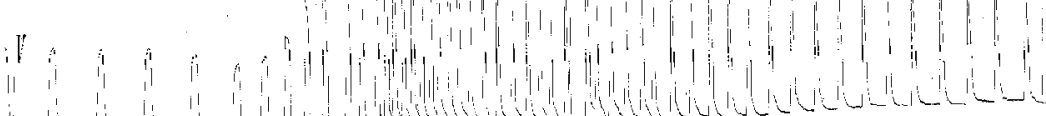




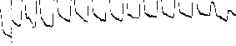
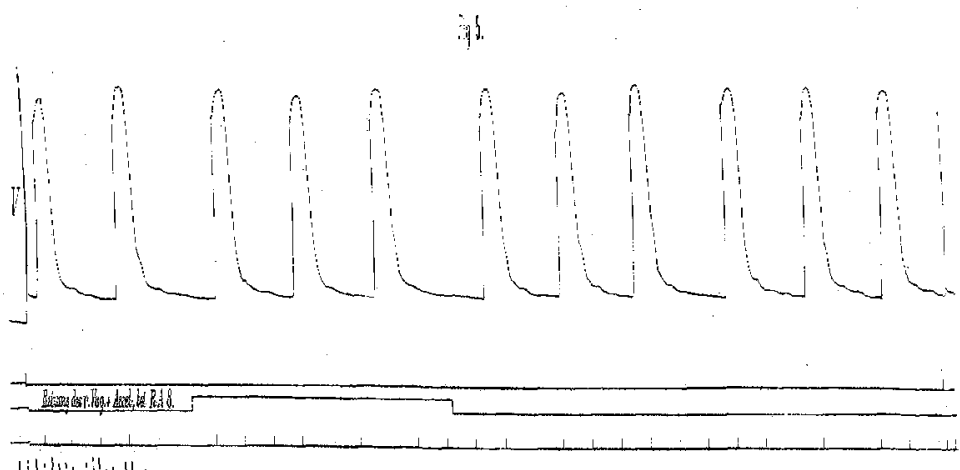

stom the

(1)

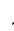
a

lin

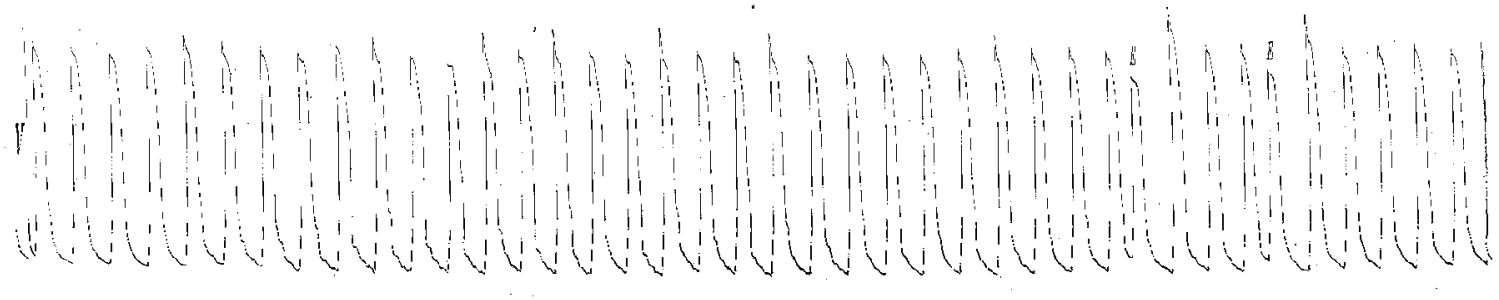

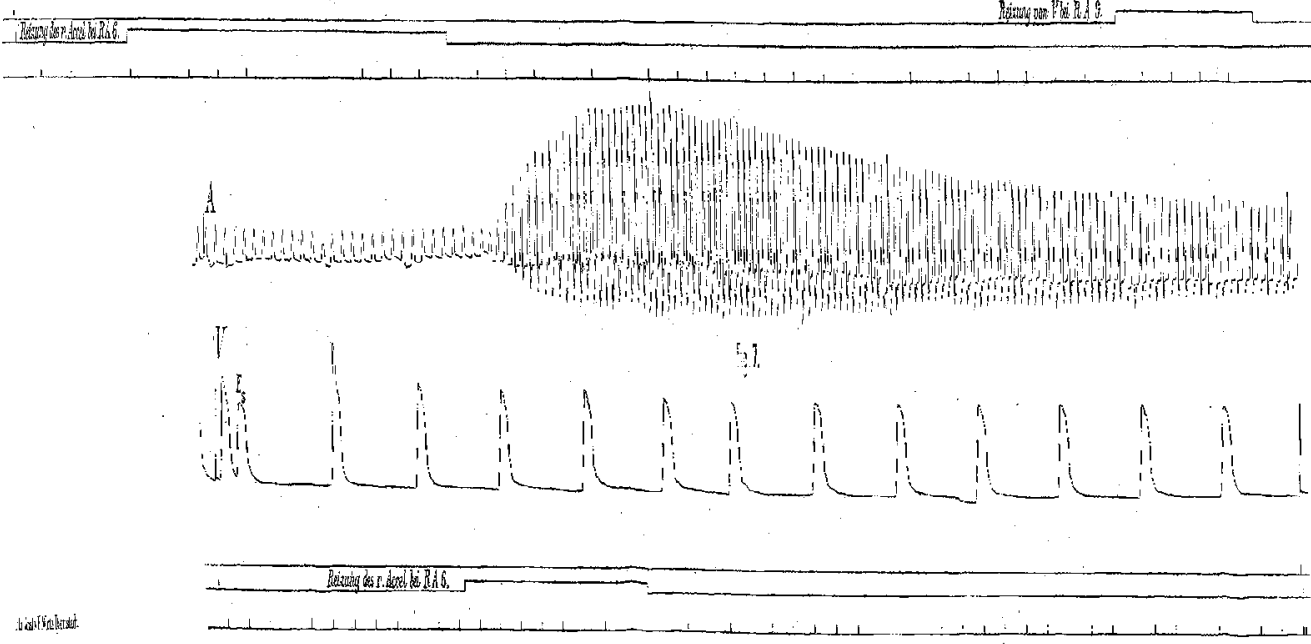




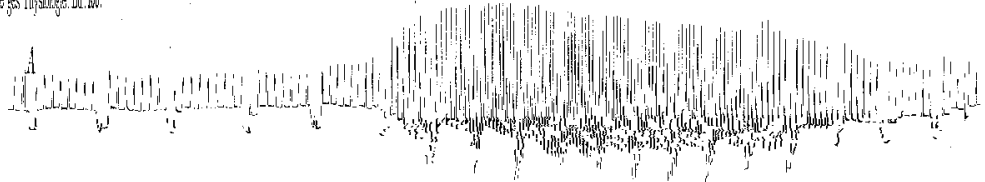

ich

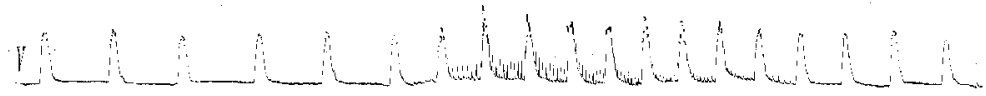

\section{Iftegedn Dol III}

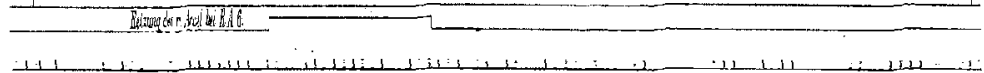

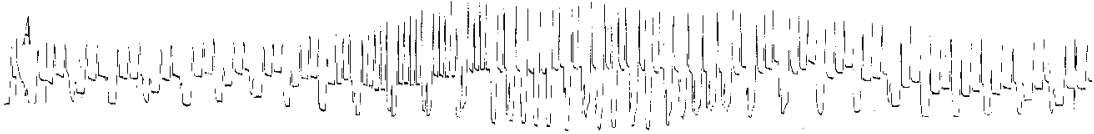
6

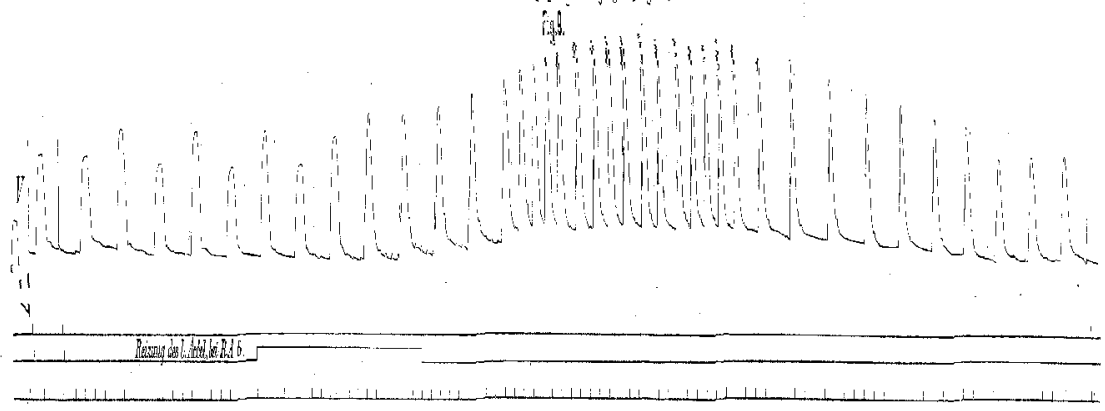

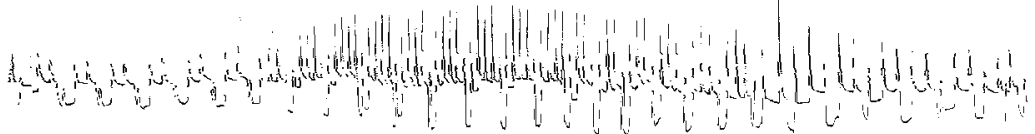

ing
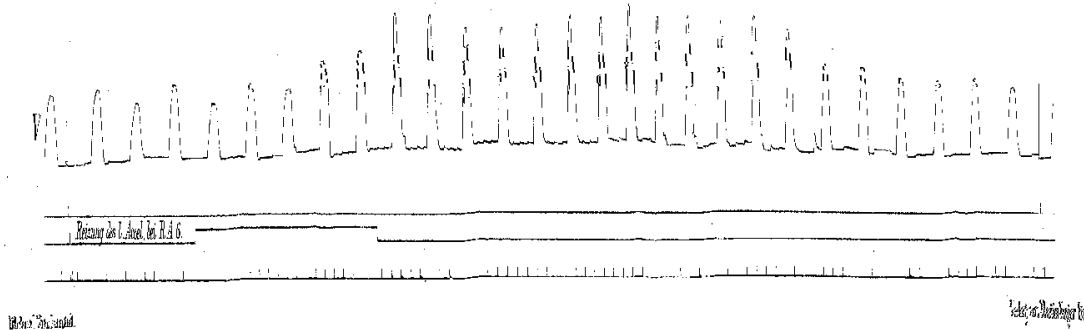


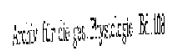
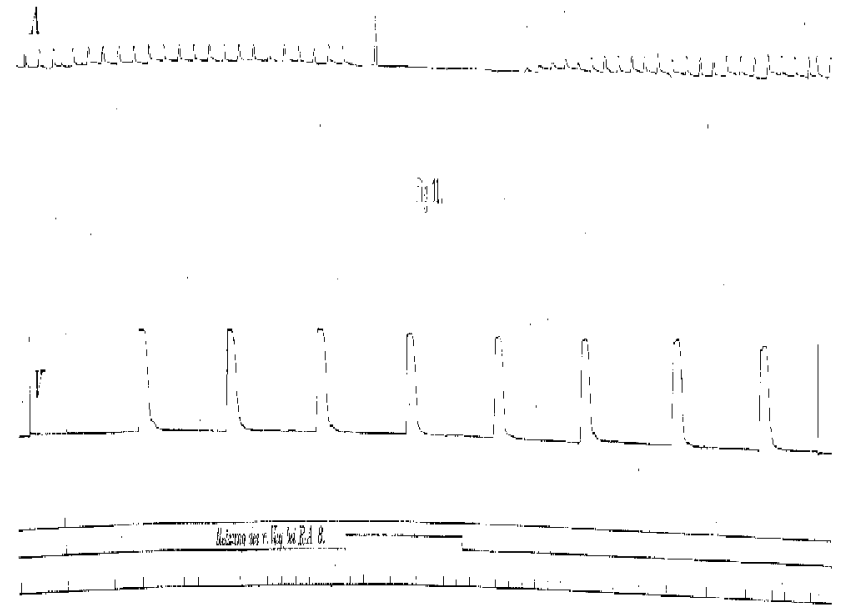

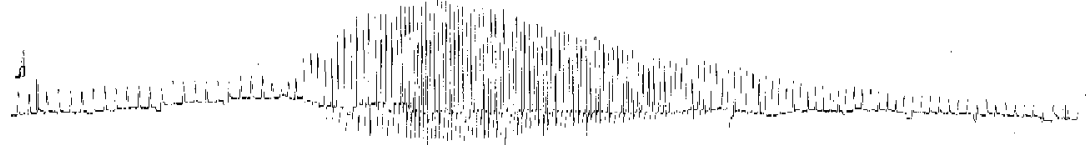

I.12

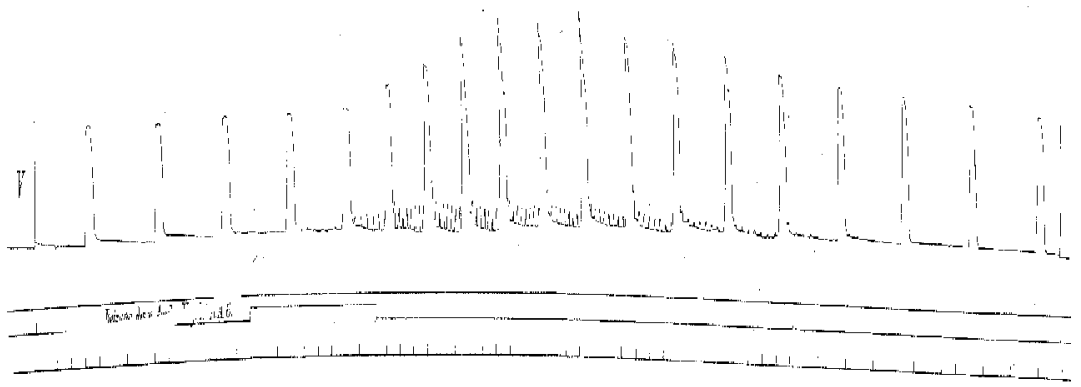

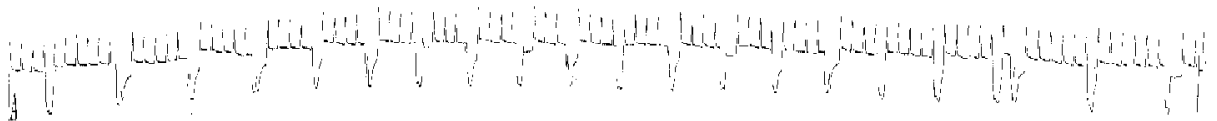
[it:?

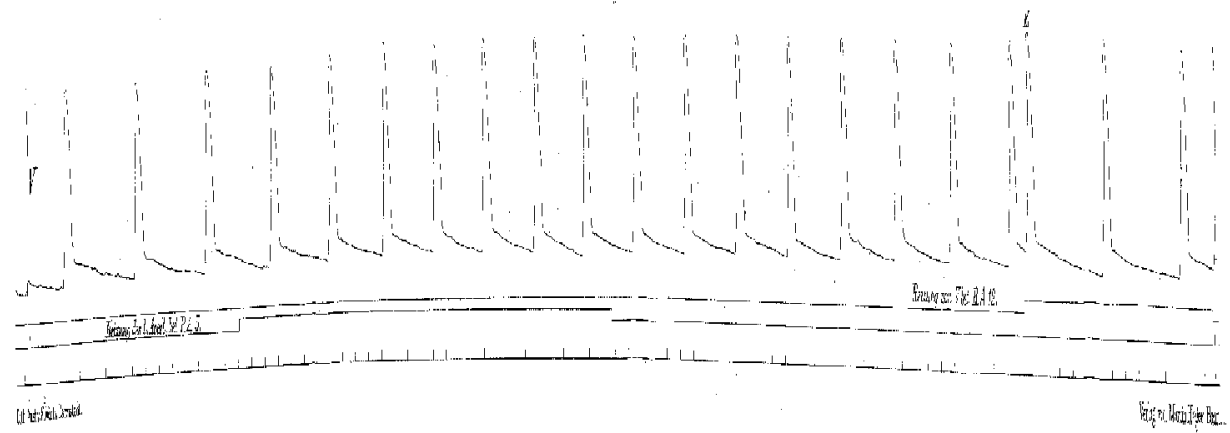


$\sqrt{1}$

Fig. 14.
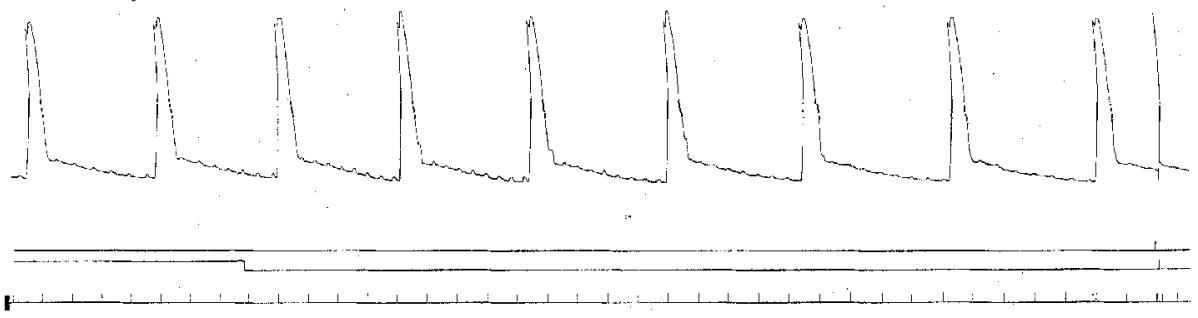

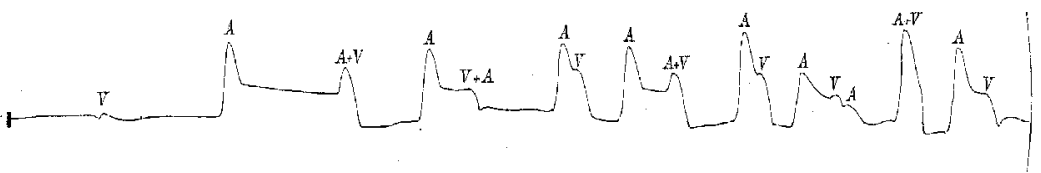

Fig. 15.

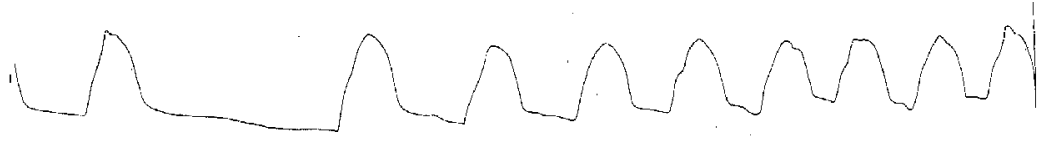

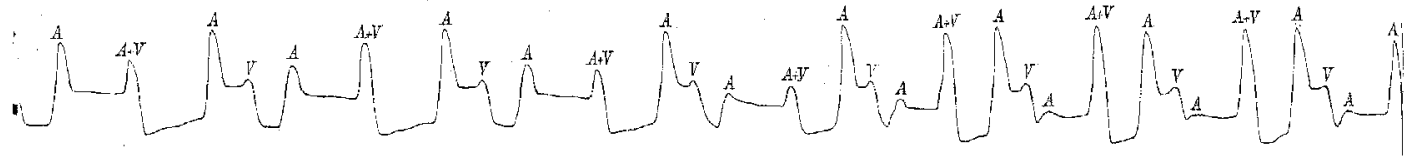

Fig. 16 .

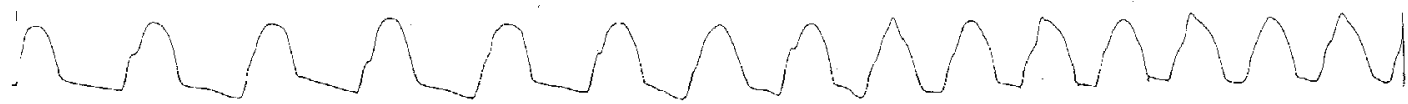

14

Fig. 17.

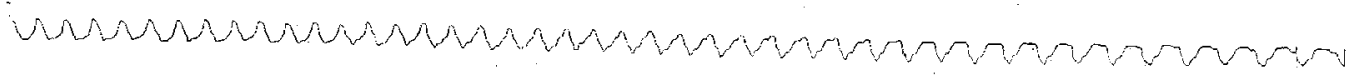

